\title{
METMAVI-VIth International Workshop on Spatio-Temporal Modelling
}

\author{
Leonhard Held*,1, Raquel Menezes ${ }^{2}$, and Inês Sousa ${ }^{2}$ \\ ${ }^{1}$ Institute of Social and Preventive Medicine, University of Zurich, Hirschengraben 84, 8001 Zurich, \\ Switzerland \\ ${ }^{2}$ Department of Mathematics and Applications, Minho University, Guimarães, Portugal
}

Received 20 January 2014; revised 20 January 2014; accepted 21 January 2014

The VIth International Workshop on Spatio-Temporal Modelling (METMAVI) took place in Guimarães, Portugal, from 12 to 14 September 2012. The meeting was organized by Raquel Menezes as chair of the Local Organizing Committee. METMA stands for a series of workshops specifically devoted to recent developments in spatiotemporal statistics since 2001, and it has been traditionally announced as a satellite conference of The International Environmetrics Society (TIES). The program of the 2012 workshop had the specificity of highlighting applications related to Health and Environmental Sciences, with particular focus on research questions from epidemiology. More than 100 research scientists from 15 different countries (see Figure 1) attended the invited and

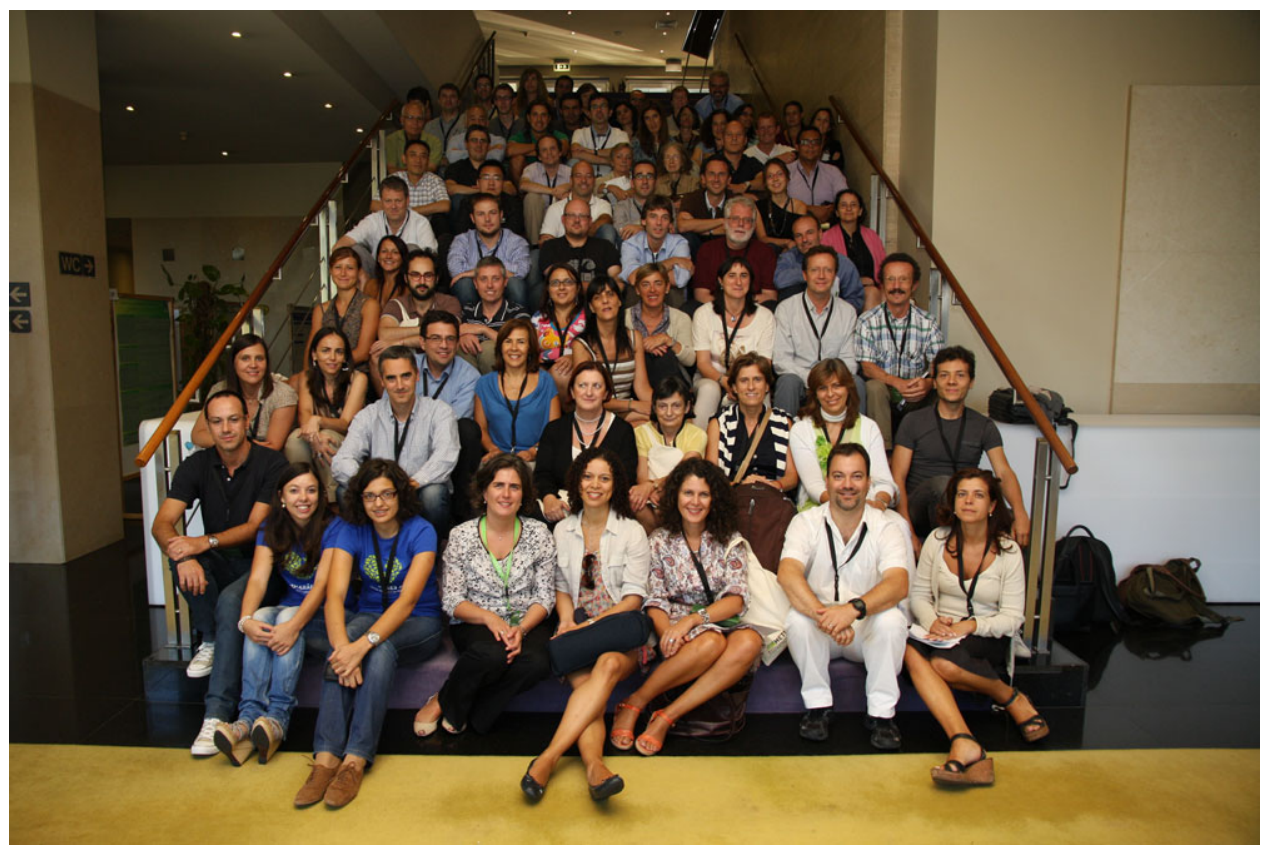

Figure 1 Group photograph taken at the METMAVI in Guimarães.

\footnotetext{
*Corresponding author: e-mail: held@ifspm.uzh.ch, Phone: +41-44-6344640
} 
contributed sessions of the workshop and the fruitful discussions afterwards. The program schedule, with no parallel sessions, facilitated the exchange of ideas and experiences.

Apart from the scientific program of METMAVI, we should not forget the historic atmosphere of Guimarães, making it a perfect venue for a scientific meeting, where reflection and debate could be inspired by the city's legacy. Guimarães, the founding location of the Portuguese Nation, was declared a World Heritage Site in 2001 by the UNESCO, and it was nominated the European Capital of Culture in 2012 .

In this special issue, the main contributions, related to spatio-temporal epidemiology, made at METMAVI are summarized. Geilhufe et al. discuss the impact of movement network data on modeling the spatiotemporal spread of infectious diseases. Their analysis is based on weekly reported counts on influenza A infections in Northern Norway, where information on human travel was compiled from data sources on travel by car, airplanes, and ferries. Etxeberria et al. present new methodology to estimate and predict risk in spatio-temporal disease mapping models. Papoila et al. present a thorough analysis of the spatial variation in stomach cancer risk in Southern Portugal in the period of 1998-2006. Duarte et al. discuss structured additive regression (STAR) models on a spatio-temporal analysis of the age of menarche and the age of menopause in women of a Portuguese cancer registry. Finally, spatio-temporal extensions of SIR models are described by Yu et al., with an application to model a dengue fever outbreak in Kaohsiung City, the second largest city in Taiwan.

We would like to express our gratitude to all referees who made the edition of this special issue possible. We are especially grateful to the Editorial Board of the Biometrical Journal for their support. We hope that these contributions will further enhance the current interest in statistical methods in spatiotemporal epidemiology.

Leonhard Held, Raquel Menezes, Inês Sousa Guest Editors 